\title{
Research on the Face Recognition and Modeling Technology and the Applications on Computer 3D Animation Design
}

\author{
Honglin Huang \\ Guangzhou International Economics College, Guangdong 510540, China \\ honglinhuang1@126.com
}

Keywords: Face Recognition, Modeling, 3D Animation, Design, Application, Computer Tools.

\begin{abstract}
In this paper, we conduct research on the face recognition and modeling technology and the applications on computer 3D animation design. Animation is a human creation so far one of the more perfect art form. It not only gives all inanimate objects to life, but also can make the inanimate objects become conscious biology and movement as the movement of the core problem is the animation in animation design. Movement and the forms of life rhythm is things, rhythm grasp of artistic creation is with the problem of universal law, which is the important factor of successful animated film. With this basis, we propose the novel paradigm for the $3 \mathrm{D}$ animation design that is meaningful.
\end{abstract}

\section{Introduction}

Modern society is based on information technology as the foundation of general social development, contracted, fashionable become modern society people's pursuit of primary fashion, contemporary art elements also shows the current era, with the digital and networked, contemporary art no matter from the aspects of text or image, has extremely high abstractness, the use of symbols is one of the most commonly used in the contemporary art performance, will use the art processing complicated things and abstract into works of art that is the main style of contemporary art [1-2].

Animation character charm can through the animation role modelling the role of design principle action performance of comprehensive expression comes out. A character's personality charm is, as it were, a character design is the key to success and market reactions of animated film will eventually induction to the role of work's popularity. Graphic design and animation design need to be by means of mobilization and control of visual elements to visual perception, causing the audience recognition, finally realizes the information transmission from person to person and in the world after entering the information age, the design elements in the process of the planning, production, convey, unavoidably cause different media penetrate each other in the way of information organization, and the penetration provides a visual graphic design style in the field of animation extension of space.

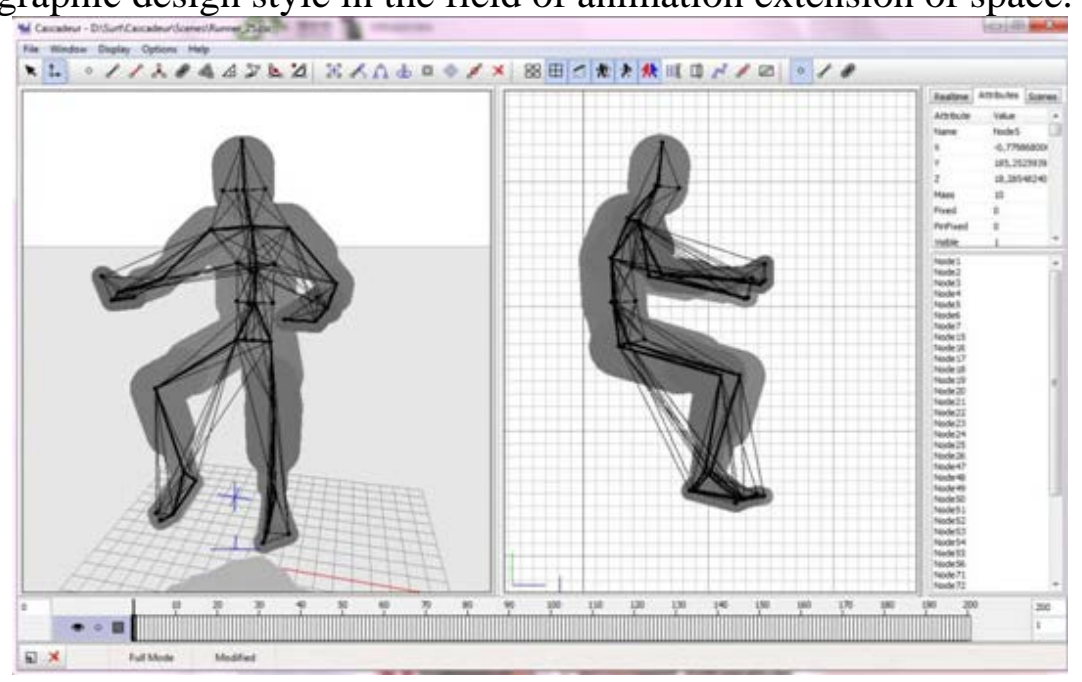

Figure 1. The Demonstration of the 3D Animation Design 
As illustrated in the above figure one, we show the sample 3D animation design pattern. As is the core aspect, we summarized the characterstics of the design pattern as follows. (1) Art is for refining and sublimation of social life, which contains a huge social and cultural value, from the works of art, people can feel the mainstream of that contemporary social thought, and produces aesthetic feelings through art appreciation, through the comments on the works of art to express thoughts, this is just the embodiment of artistic spirit. (2) Contemporary art and animation design, animation design workers must pay attention to in the design process regularly use some kind of artistic techniques, in order to show the art of animation design value to make animation design animation film and animation works around the creation, the basis of making animation design as part of the contemporary art.

\section{Our Proposed Algorithm}

Image Representation. Compressed sensing as a new signal acquisition and compression method has been widely used in the image processing field, which is to avoid the high-speed sampling and compression caused by the waste of large amounts of data, and reduce the acquisition and subsequent transmission and store the data of pressure. It breaks through the limit of specific principle of Nyquist sampling and there are strict mathematical model, the sampling of signal into information collection, pay more attention to the internal structure of the signal. It can be used to collect data to huge large probability to restore the original signal, this make it have been able to satisfy people's need for access to information in reality. Traditional compression perception theory is that as long as the signal in a transform domain with sparse, via a small amount of the original signal in the observational domain projection, with the nonlinear iterative method of large probability of recovering the original signal.

$$
x=\sum_{i=1}^{N} \theta_{i} \varphi_{i}
$$

Wavelet modulus maxima method using binary convolution wavelet for image of the modulus and phase diagram, by the Lipschitz index to depict the singularity of signal, signal and noise in different scales by using the value on the graph, the noise using the modulus maxima principle, and then uses the iterative implementation of alternating projection by reconstructing wavelet coefficients modulus maxima points, this method of large amount of calculation [3].

Face Modeling. Face as a person is the most important outside characteristic, in the process of the interpersonal communication plays a very important role. It can be many basic nonverbal message to strengthen understand or to express the emotion. Realistic computer facial animation is one of the most basic problems in computer graphics field.

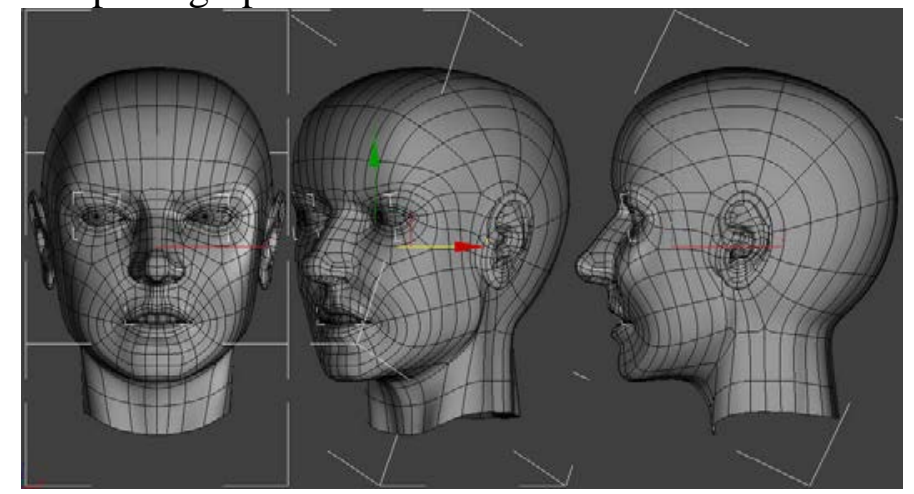

Figure 2. The Demonstration of the Face Modelling Procedures

It is no good describe the 3D model of human face. In addition, the face recognition also involves image processing, computer vision, pattern recognition and neural network subject, and also closely associated with the degree of the understanding of the human brain that can be generally organized as the following aspects. (1) Parameterized: can form a specific face model by adjusting parameters. (2) Simplicity: medium model of vertices, balance between accuracy and complexity. (3) Visualization: describe face basic structure accurately, and can make some expressions.

Face parameter model to consider two parameters, respectively is face structure parameters and the facial animation parameters. Face structure parameters are used to describe the structure of the 
human face information, such as the size of the face, facial features, location, etc., through the adjustment of parameters can achieve different specific face as facial animation parameters are used to describe the movement of the facial organs to produce a variety of facial expression animation. Accordingly, we could summarize the face modelling procedures as the follows.

$>$ In computer facial animation, facial expressions, the details of the small changes such as the illumination change, face skin color moments, wrinkles, etc., can greatly enhance realism to the facial model can more nonverbal message at the same time.

$>$ Face model to make use of texture image rendering model can greatly enhance the sense of the reality, however, texture image reflects only face the light conditions when being filmed, it usually introduced are not real effect.

$>$ Computer facial animation is the purpose of through the provision of a complete, the concise facial animation process control model and the control deformation between the facial model, simulate real facial movements and expressions.

Face Recognition. Face recognition is a kind of image analysis and the understanding of the most successful applications, because of its in the commercial, security, authentication, law enforcement, and many other aspects of the widely used, and the feasibility of face recognition technology for more than 30 years of research make it get more and more attention, and become a vibrant research areas.

$3 \mathrm{D}$ image contains information within the scope of the whole face space, can be largely overcome the effects of these factors on the effect of face recognition. With the continuous development of 3D technology, face recognition based on 3D face imaging problems also gradually become the research hot spot. Face recognition system is only at the laboratory stage, is a certain distance from the practical application of themselves and as the key reason: the deterrent rate system algorithm is complex, long operation time and does not meet the requirement of real-time to achieve the real-time property of the system error recognition rate is relatively high [4].

$$
\min \varepsilon(W)=\sum_{i=1}^{N}\left|x_{i}-\sum_{j=1}^{k} w_{i j} x_{i j}\right|^{2}
$$

Then the basic subspace analysis method has attracted widespread attention, become one of the mainstream of the current face recognition methods. The idea of subspace analysis is based on certain performance goals to look for a linear or non-linear space transformation the original information data compression to a low-dimensional subspace, more compact, and the data in the subspace distribution for better description of data provides a means of the complexity of the calculation has been reduced.

Computer 3D Animation Design. Binding technology is a key technology to make 3D character animation, main function is the role model for assembly, the animators can control characters and the animation like a puppet, as works by early perceptual thinking into a logical thinking and that specific content involved in object space coordinates, hierarchy, rotating order, bone dynamics, application property control, expression and scripts, and so on can master the binding technology is knocking on the door on the three dimensional animation.

The success of any animated works, in addition to have a good story, and let you'll never forget the role of modelling. While the latter is more important and the audience will often remember the film because of the piercing modelling. According to the preliminary design, animation is made by related software, production process is: modeling, materials, lighting, animation, rendering, etc., this is the three dimensional animation production characteristics that can be expressed as follows.

$>$ Texture, material or material quality of a material, it is on the surface properties of the model gives vivid, concrete embodiment in the object's color while transparency as the luminosity reflective strength, illuminations and roughness etc. related characteristics.

$>$ The lights. The lights on the 3D software generally have Omni lights and direction. Lighting plays a lighting scene, cast shadows, and the function of add atmosphere. Usually uses three light setup method: an advocate the lamp, a lamp, and a back light.

$>$ The camera movement. According to the principle of photography in the three dimensional animation software using the camera tool, achieve continuity design lens effect. The stability of the picture, fluency is to use the camera, the first thing to do. 
The animation. According to the continuity and motion design, apply the modelling has been designed in 3D animation software to produce small animation section. Action and the change of the image by key frames that 3D software will mostly animation information represented by general and ordinary primary related animation curve.

In the modelling design practice basic skills, deal with the requirements of every part of the whole animation into consideration, whether it is painting or collected material should have more clear goals and pertinence, and according to the different style of animation for the modelling of related training.

Animation Design Characteristics. Animation is a complex art that covers a lot of the design knowledge, and the color is such a moment is closely related to art but also with the elements of core animation together. The role of color in animation design is various and it can reflect the character, the character image that also can show conflict, highlighting the style of work and effect.

Animation design is the foundation of the animation, including animation character design, scene design, music, and other links. Animation design according to its work characteristics belong to a part of art design, animation design forms and like art and film and television works, to say the animation design is part of the contemporary art, to discuss the relationship between contemporary art and basic animation design, animation design development of rich contemporary art, it is very necessary.

In an animation work, through the use of the emotional characteristics of color to lead the viewer's emotional experience, to mobilize the viewer's emotions, can better shape bright and round the role of the image, can create a suitable scene atmosphere to foil performance main body, strengthen the plot ups and downs that can help the viewer understand the theme of works, etc. In process of animation design, animation, to seize the animation role modelling design personnel can highlight some of the outstanding personality, by using basic exaggerated art gimmick, to shape every animated characters become role with distinct personality characteristic can fully highlights its distinctive characteristics, so as to attract the audience's emotions in the animation, the resonance of the audience.

\section{Conclusions}

In this paper, we conduct research on the face recognition and the basic modeling technology and the applications on computer 3D animation design. Art is summed up in the work and life of thought and combination of beauty, art style is very rich, literature, painting, sculpture and music are the reflection of art in real life. Animation role is a cartoon story to promote the main undertaker, the audience to judge the basic value of an animated character can not only rely on external visual effect. Animated characters can be accepted by the public, the key lies in whether it has the fresh vitality, the vitality of sense must be determined by the character's personality. Our research combines the basic features of the related techniques to propose the 3D animation design pattern that is innovative.

\section{References}

[1] Cleeren, Gertjan, et al. "Role of 3D animation in periodontal patient education: a randomized controlled trial." Journal of clinical periodontology 41.1 (2014): 38-45.

[2] Schultz, Julia A., and Thomas Martin. "Function of pretribosphenic and tribosphenic mammalian molars inferred from 3D animation." Naturwissenschaften 101.10 (2014): 771-781.

[3] Al-Qassabi, Hamed, and Hosam Al-Samarraie. "Applying Gagne’s Nine Events in the Design of an Interactive eBook to Learn 3D Animation." Advances in Computing 3.3 (2013): 60-72.

[4] Wang, Shen. "Motions Blur Effects in the Process of Three-Dimensional Animation Technology Research." Applied Mechanics and Materials. Vol. 687. Trans Tech Publications, 2014. 